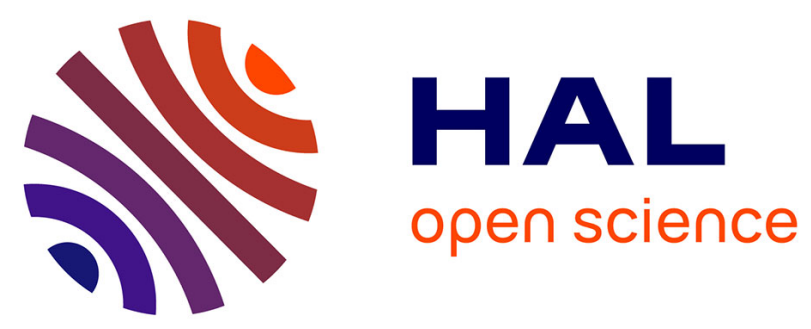

\title{
ELECTROREFLECTANCE SPECTROSCOPY IN THE STUDY OF METAL-ELECTROLYTE INTERFACES
}

\author{
D. Kolb
}

\section{To cite this version:}

D. Kolb. ELECTROREFLECTANCE SPECTROSCOPY IN THE STUDY OF METALELECTROLYTE INTERFACES. Journal de Physique Colloques, 1983, 44 (C10), pp.C10-137-C10146. 10.1051/jphyscol:19831029 . jpa-00223486

\section{HAL Id: jpa-00223486 https://hal.science/jpa-00223486}

Submitted on 1 Jan 1983

HAL is a multi-disciplinary open access archive for the deposit and dissemination of scientific research documents, whether they are published or not. The documents may come from teaching and research institutions in France or abroad, or from public or private research centers.
L'archive ouverte pluridisciplinaire HAL, est destinée au dépôt et à la diffusion de documents scientifiques de niveau recherche, publiés ou non, émanant des établissements d'enseignement et de recherche français ou étrangers, des laboratoires publics ou privés. 


\title{
ELECTROREFLECTANCE SPECTROSCOPY IN THE STUDY OF METAL-ELECTROLYTE
}

\section{INTERFACES}

\author{
D.M. Kolb \\ Fritz-Haber-Institut der Max-Planck-Gesellschaft, Earadayweg 4-6, \\ 1000 Berlin 33, F.R.G.
}

\begin{abstract}
Résumé - L'électroréflectance des surfaces monocristallines de $\mathrm{Cu}$, Ag et $\mathrm{Au}$ en contact avec une solution aqueuse est brièvement passée en revue et discutée. On montre que les états de surface à l'interface métalélectrolyte contribuent de façon significative à l'effet global d'électroréflectance et peuvent fournir des renseignements sur la distribution du champ électrique à 1 'intérieur de la double couche électrochimique. L'électroréflectance infrarouge peut être utilisée pour détecter directement certaines contributions des composants de la double couche. Finalement le rôle de l'optique non-locale pour l'interprétation des spectres d'électroréflectance est mis en valeur.
\end{abstract}

Abstract - Electroreflectance of $\mathrm{Cu}, \mathrm{Ag}$ and $\mathrm{Au}$ single crystal surfaces in contact with an aqueous electrolyte is briefly reviewed and discussed. It is shown that surface states at the metal-electrolyte interface contribute significantiy to the overall electroreflectance effect, and they can provide information on the electric field distribution inside the electrochemica? double layer. Infrared electroreflectance may be used to detect contributions from the double layer constituents directly. Finaliy, the role of non-local optics for interpretation of electroreflectance spectra is emphasized.

\section{1 - Introduction}

Classical electrochemical techniques which are based on charge and potential measurements, yield in essence a thermodynamic description of the electrochemical interface $/ 1 \%$. However, in order to derive a microscopic picture, information regarding the electronic and geometric structure of the double layer region is very important. In this respect, reflectance spectroscopy promises a better understanding by investigation of the optical and hence electronic properties of the bare and adsorbate-covered electrode surfaces. It is applicable in-situ, specific at an atomic or molecular level, and sensitive enough to characterize in detail electrode-electrolyte interfaces. Among the various spectroscopic techniques, electroreflectance (ER) was regarded as the most appropriate tool for investigation of the metal-electrolyte interface. In the absence of any electrochemical reaction, this interface behaves like a capacitor which can be charged or discharged by appropriate potential variations. Since the double layer capacity ranges between 20 and $100 \mu \mathrm{Fcm}^{-2}$ and the potential region for double layer charging for a metal like silver is about $1 \mathrm{~V}$, large surface charges (say, $20 \mu \mathrm{Ccm}^{-2}$, corresponding to about 0.1 electron per surface atom!) and high electric fields $\left(\sim 10^{7} \mathrm{Vcm}^{-1}\right)$ can be obtained with ease and their values modulated by simple potential modulation $/ 2 \%$. Such a potential variation in the double layer charging region has been found to cause a noticeable change in the reflectance of the metal-electrolyte interface $/ 3 \%$. This change in reflectance with electrode potential is called electroreflectance (ER). In contrast to semiconductor ER where the static or low frequency electric field penetrates into the bulk several thousand Angstroms, thus probing the bulk band structure $/ 4 /$, the perturbing electric field is screened within the first atomic layer of the metal because of the high charge carrier density (the Thomas-Fermi screening 


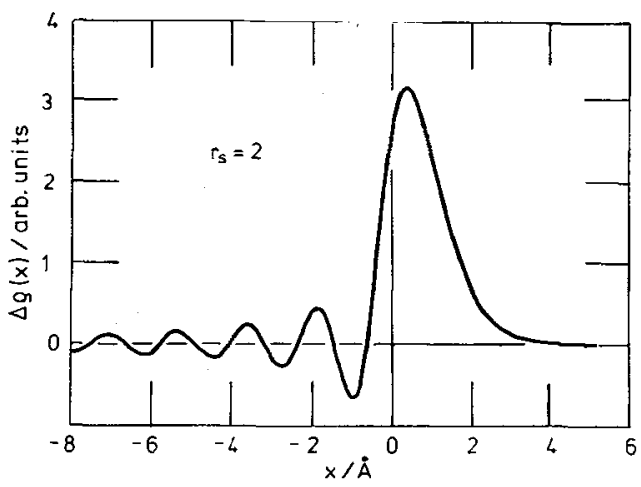

Fig. 1 - Surface charge density $\Delta \rho$ of a metal, induced by a weak external electric field. $x=0$ denotes the edge of the positivecharge background. After ref. /5/. length is about $0.5 \%$ ). Hence ER for metals should be extremeiy surface sensitive. This is demonstrated in Fig. 1 , where for a jellium metal, the surface charge density induced by a weak externa? electric field is shown $/ 5 /$. In the following, various contributions to meta? ER are briefly discussed, restricting the examples to studies of single crystal surfaces only.

\section{2 - ER Spectra of $\mathrm{Cu}$ and Ag Surfaces}

Early experiments were performed with polycrystalline surfaces $/ 6 /$. The results, mainly for $\mathrm{Ag}$ and $\mathrm{Au}$, were interpreted in terms of the "free-electron" model developed by Hansen $/ 7,8 /$ and refined by McIntyre $19 /$. This model assumes that the change in the free-electron concentration at the surface, induced by the potential change, is mainly responsible for the observed effect. Bound electrons are taken to be perfectly screened by the free electrons, hence they do not respond to the applied electric field. The free-electron model has proven to reproduce the ma in features of an ER spectrum, especially for polycrystalline surfaces (e.g., the peak at $3.9 \mathrm{eV}$ for $\mathrm{Ag}$ and $2.5 \mathrm{eV}$ for $\mathrm{Au}$ ); however, these gross features in $\Delta R / R$ arise mainly from the $1 / R$ effect, which is always present for metals with rapidiy varying reflectivity. Many details in the spectra could not be explained by this simple model, indicating that there are other sources which contribute significantly to the ER and which had been neglected so far. The marked differences which are observed for the various crystallographic faces of one and the same metal strongly suggested that the bound electrons do feel the modulating potential $/ 10,11 /$. This implies that the ER spectra of single crystal surfaces contain information about the band structure in the surface region and its dependence on the electrode potential.

The failure of the free-electron model to reproduce even pronounced effects in ER is demonstrated in Fig. $2 / 12 /$. Here the ER spectra for Cu(111) on mica are shown for $s-$ and $p$ - polarized light at $45^{\circ}$. Note that the sign of $\Delta R / R$ is such that a positive value refers always to a reflectivity increase with positively going potential. The pronounced peaks in the spectra at $2.2 \mathrm{eV}$, at the onset of the interband transition, cannot be reproduced by the free-electron model alone. However, satisfactory agreement between theory and experiment was obtained when a shift of the interband transition energy with electrode potential was taken into account. This shift amounts to about $0.1 \mathrm{eV}$ for a $0.5 \mathrm{~V}$ potential change /12/. The experiment cannot yet answer the question whether this change in band structure is solely caused by the potential influence or whether the surface region already in the absence of an external field possesses an electronic structure which differs from that of the bulk because of surface reconstruction.

Besides an actual change in the electronic states at the surface due to the very high electric field which leads to a modulation of the interband contribution to $E R$, there can be a noticeable modulation of the interband transition proper. As was pointed out by Lynch $/ 13 /$, this may occur via field-induced "indirect" interband transitions. Because of the high field strength attainable at metal-electrolyte interfaces, the uncertainty in the wave vector parallel to the applied field can be appreciably large (up to $0.1 \AA^{-1}$ ), hence allowing for $k$-conserving interband transitions over a larger variety of final states than in the absence of the field $/ 13 /$. 


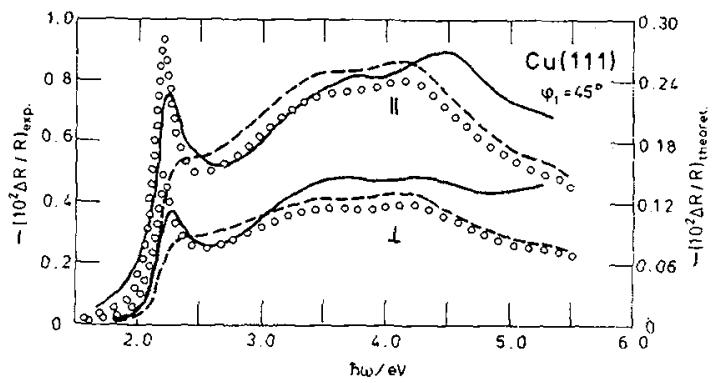

Fig. 2 - ER spectra of Cu(111) at $45^{\circ}$ and s- and p-polarized light. (-) Experimental curves for $\Delta U=500 \mathrm{mV} .(--)$

Calculated by the free-electron model. (oooo) Adding to the free-electron model a $0.1 \mathrm{eV}$ shift with potential of the interband transition. After ref. /12/.

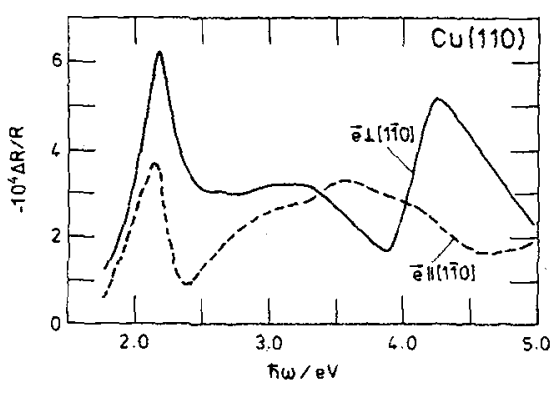

Fig. 3 - Normal-incidence ER spectra of $\mathrm{Cu}(110)$ in $1 \mathrm{~N} \mathrm{H}_{2} \mathrm{SO}_{4}$ for two different directions of the electric vector of the Iinearly polarized light. $\mathrm{U}_{0}=-0.3 \mathrm{~V}$ vs. SCE. $\Delta \mathrm{U}_{\rho p}=100 \mathrm{mV}$. After ref. /12/.

In Fig. 3 the normal-incidence ER spectra for $\mathrm{Cu}(110)$ are shown for two different crystallographic directions. This polarization anisotropy, which was first reported for $\mathrm{Ag}(110)$ by Furtak and Lynch $/ 10,14,15 /$, reflects the two-fold symmetry of the surface and has been observed for the (110) faces of $\mathrm{Cu} / 12 / \mathrm{Ag}$ /10/ and $A u / 16,17 /$. It demonstrates again that band structure effects and crystal symmetries influence the $E R$ effect. The dependence of the polarization anisotropy on the surface crystallographic direction is shown in Fig. 4 for $\mathrm{Ag}$ (110) as the electric vector of the linearly polarized light is rotated by $360^{\circ}$. It is interesting to note that for $C u$ and $A g, \Delta R / R$ is largest for $\vec{e} \| l[001]$ (i.e., across the atomic rails) and smallest for $\vec{e} \|[110]$ (i.e., along the densely packed atomic rails) while the opposite behavior is found for Au(110) $/ 16,17 /$. Secondly, we find that the polarization anisotropy is found over a wide range of photon energy and not at all limited to the interband transition region $/ 18 \%$. The potential dependence of the polarization anisotropy is reproduced in Fig. 5 for $A g(110)$, where the highest anisotropy is found at potentials positive of the potential of zero charge (pzc: $-1.0 \mathrm{~V}$ ), while the anisotropy tends to disappear rather quickly for $\mathrm{Ag}(110)$ as the surface becomes negatively charged $/ 15 /$.
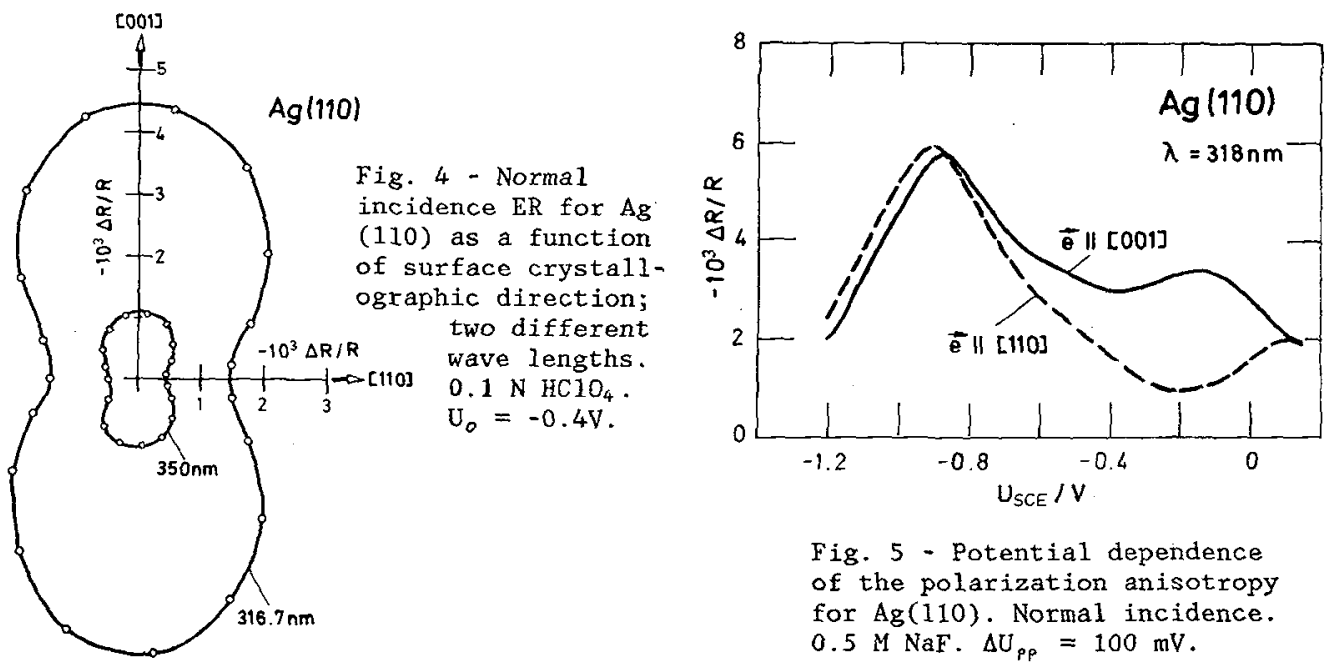

Fig. 5 - Potential dependence of the polarization anisotropy for $\mathrm{Ag}(110)$. Normal incidence. $0.5 \mathrm{M} \mathrm{NaF} . \Delta U_{p p}=100 \mathrm{mV}$. 
Finally we briefly discuss how the ER effects for various crystallographic surfaces compare $/ 19,20 \%$. This was done in the case of Cu by the use of a single crystal cylinder whose axis was parallel to [110]. The various high and low index faces were then studied optically by the appropriate rotation of the cylinder. The results for two different wave lengths are shown in Fig. 6. While the anisotropy is largest for the (110) and adjacent faces, the largest effect in $\Delta R / R$ is clearly observed for the (113) face. For $\lambda=570 \mathrm{~nm}$, the polarization
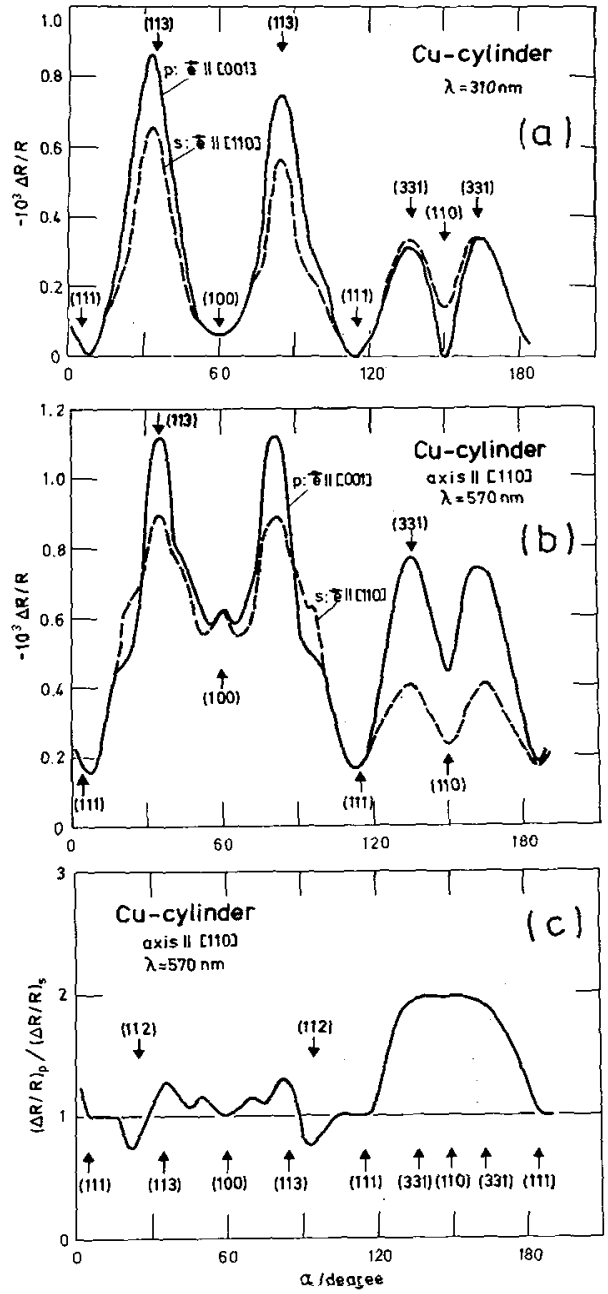

Fig. 6 - Normal-incidence ER for $\mathrm{Cu}$ as a function of crystallographic orientation $(a, b)$ and polarization anisotxopy (c). $1 \mathrm{~N} \mathrm{H}_{2} \mathrm{SO}_{4} \cdot \mathrm{U}_{0}=-0.3 \mathrm{~V} \cdot \Delta U_{p P}=100 \mathrm{mV}$. After ref. $/ 19,20 /$.

anisotropy as it cannot account for only source for the polarization observed potential or wave length dependencies of the anisotropy. For example, it has been shown that surface states at the metal-electrolyte interface can play a significant role for

(110) Since the high index faces of the type (hhl) ( $h \geqslant 3)$ can be considered as stepped surfaces with (110) terraces, it seems that the anisotropy is a characteristic of the (110) surface proper rather than of a surface of two-fold symmetry in general.

The results of Fig. 6 seem to indicate that the more open the surface structure is, the larger the ER signal is. $\triangle R / R$ is in general found smallest for the most densely packed (111) face. This has been tentatively explained by a smoothing effect for the more open surfaces, which causes larger changes in the optical polarizability of the surface layer with potential $/ 12,18 /$. The electron density contours for an open surface should reproduce the wavy structure of the positive background parallel to the surface. However, as was pointed out by Smoluchowski /21/, the smoothing effect tends to reduce any structure in the density profile parallel to the surface by accumulating negative charge in the valleys. Charging the metal electrode surface will affect the electron density distribution not only perpendicular to the surface but also parallel to it. While for a densely packed surface or surface direction, the electron tail may be just pushed outward with negative charging without much change in shape, it may change the density contours at a more open surface or surface direction by changing the degree of smoothness. At negative potentials the increase of charge should smooth out the spatial variation of the electron density parallel to the surface, while at positive potentials the positive background contours should appear more strongly. Hence, the potential induced change in the optical polarizability of a surface should be larger for more open surface structures, causing larger $\Delta R / R$ values as found in the experiment. This effect, however, does not seem to be the only source for the polarization interface can play a significant role for
inter ample, it has been shown that 
the observation of polarization anisotropy for low-symmetry surfaces, as will be discussed in the following.

\section{3 - Surface States at the Metal-Electrolyte Interface}

The role of electronic surface states in electroreflectance was first pointed out by Ho, Harmon and Liu /22/. When calculating the two-dimensional (surface) band structure of a metal, i.e., the projection of the bulk band structure onto a plane parallel to the surface, energy gaps are found in certain crystallographic directions. In a self-consistent pseudo-potential calculation for $A g(110)$, Ho et a). /22/ have shown that surface states exist in these energy gaps, which are split off the volume states at the upper and lower band edge of the gap. Such a situation is schematically shown for $\mathrm{Ag}(100)$ in Fig. 7 /23/. The surface states $A$ and $B$ are strongly localized at the surface, and rapidly decay along the surface normal. As a consequence, the energetic position of the surface states will depend on an applied external field in a way which is distinctly different from that of the bulk states. Hence, optical transitions from the bulk states to a surface state should be influenced in their energy by the electrode potential. This dependency can be used to detect and identify surface states at the metal-electrolyte interface. In a careful study $/ 23,24 /$ of the ER spectra of $\mathrm{Ag}(100)$, two spectral features were indeed observed, the energetic positions of which varied strongly with the electrode prtential (see Fig. 8).

When the electrode potential of a metal electrode is changed by $1 \mathrm{~V}$ positively or negatively, then the whole bulk band structure (including the Fermi level) is shifted with respect to the vacuum level by exactly I eV downward (to higher work function) or upward (towards lower work function). Since the surface states penetrate somewhat into the double layer, they experience only a certain fraction of the total potential drop across the Helmholtz layer, as the electrochemical double layer is frequently called. Hence the surface states are shifted less in energy than the bulk states. As a result, the optical transition from an occupied bulk state into an empty surface state varies with potential such that the transition energy increases as the potential moves in the positive direction. Such a behavior is noticed for the spectral features around 3 and $1 \mathrm{eV}$ in the ER spectra of $\mathrm{Ag}(100)$ (Fig. 8). A comparison of the experimentally observed transition energies and their dependence on the electrode potential (see insert of Fig. 8) with the calculation by Ho and Liu gave an almost perfect agreement, which allowed

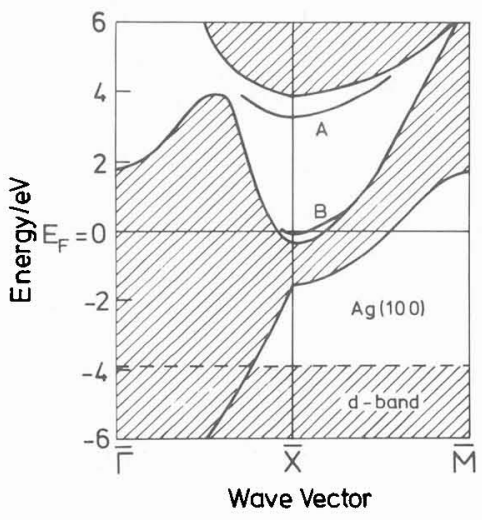

Fig. 7 - The Ag(100) projected band structure with surface states $A$ and $B$. After ref. $/ 23 /$.

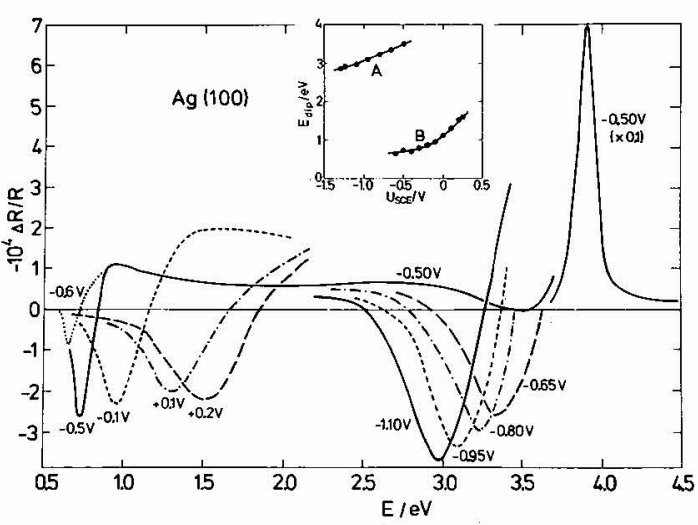

Fig. 8 - Norma1-incidence ER spectra of $\mathrm{Ag}(100)$ in $0.5 \mathrm{M} \mathrm{NaF}$ for various bias potentials. After ref. /24/. Insert: transition energies as a function of electrode potential. 
for a safe assignment of the above mentioned spectral features in the ER spectrum of $\mathrm{Ag}(100)$ to transitions from occupied bulk states into the surface states $A$ and $B$, respectively $/ 23 /$. Even the disappearance of the transition into $B$ around -0.6 $V$ is found to be in full agreement with theory which predicts the surface state $B$ to be shifted below the Fermi level at that potential $/ 23 /$.

Optical transitions into empty surface states have also been predicted for $\mathrm{Ag}(110)$ 122/. However, based on symmetry considerations, such transitions should be possible only for $\vec{e} \|[001]$, but not for $\vec{e} \|[110]$. This was indeed confirmed by experiment $/ 24 /$. The potential dependence of optical transitions into empty surface states can also be seen quite clearly, when $\Delta R / R$ is recorded as a function of bias potential for different wave lengths. Such curves are shown in Fig. 9 for $\mathrm{Ag}(110)$ for both polarization directions. Two surface state features are immediately recognized for $\vec{e} \|[[001]$, around $1000 \mathrm{~nm}$ at $0 \mathrm{~V}$ and around $800 \mathrm{~nm}$ at $-1.1 \mathrm{~V}$, and their, shift with potential is marked. No such features are found for $\overrightarrow{\mathrm{e}} \|[110]$, where the structures in $\Delta R / R$ do not shift with wave length (Fig. 9b). It is evident from a comparison of Figs. $9 a$ and $9 \mathrm{~b}$, that surface states contribute in a prominent way to the observed polarization anisotropy of $\mathrm{Ag}(110)$, as discussed in the previous section.

As the energetic position of the surface states sensitively depends on the external electric field, information about the potential distribution near the metal surface should be obtainable from an analysis of the shift in excitation energy. In our simple picture of the electrochemical interface, which we treat as a condenser with $0.3 \mathrm{~nm}$ plate distance $/ 25 /$, we assume a linear potential drop across the double layer. If we further assume that the surface states probe the region just outside the metal surface, say $0.1 \mathrm{~nm}$ away $/ 22 /$, then this would yield a relative shift of about $0.3 \mathrm{eV} / \mathrm{V}$. Such a shift has indeed been observed for Au(100) $/ 17,24,26 /$. For $\mathrm{Ag}$ single crystal surfaces, however, much higher slopes were found, up to $4 \mathrm{eV} / \mathrm{V}$ for the $\mathrm{Ag}(110)$ surface $/ 24 \%$. This indicates that the potential difference between bulk and surface states is much larger than that derived from a linear potential drop across the Helmholtz layer. The large shifts may be explained by assuming a non-linear, strongly varying potential distribution within the Helmholtz layer, caused by the microscopic structure of the interfacial water or by specifically adsorbed ions. For example, the finite size of the water dipoles may lead to

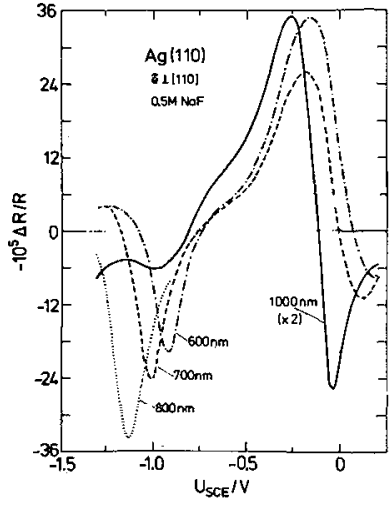

Fig. 9a - ER signals of Ag(110) in $0.5 \mathrm{M} \mathrm{NaF}$ as a function of electrode potential for various wave lengths. Normal incidence. $\overrightarrow{\mathrm{e}} \|\left[[001] \cdot \Delta \mathrm{U}_{P p}=100 \mathrm{mV}\right.$.

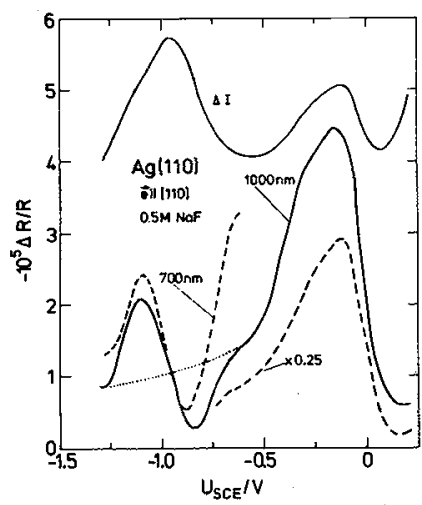

Fig. 9b - ER signals of Ag(110) in $0.5 \mathrm{M} \mathrm{NaF}$ as a function of electrode potential for two different wave lengths. Normal incidence. $\vec{e}||[110] \cdot \Delta \mathrm{U}_{\mathrm{PP}}=$ $100 \mathrm{mV} . \Delta \mathrm{I}=$ double layer charging current. pzc: $-1.0 \mathrm{~V}$. 
regions of overscreening and underscreening, as has been pointed out in corresponding model calculations $/ 27 /$. The unity slope which is found for $\mathrm{Ag}(100)$ implies that the potential difference between bulk and surface states, i.e., between metal surface and location of maximum density of surface states, is as large as the total potential drop across the Helmholtz layer. This leads to a picture with a strongly varying potential in the double layer, similar to that for specific adsorption where an "overshooting" of the potential occurs $/ 25 /$. The very high slopes of $3-4 \mathrm{eV} / \mathrm{V}$ observed for $\mathrm{Ag}(110)$, however, are difficult to rationalize in such a purely electrostatic description, and other effects, such as chemical interaction, may have to be considered in addition.

Finally, we briefly focus our attention on the line shape of the absorption band for the optical excitation into surface states. As a consequence of the modulation technique applied for detecting electroreflectance signals, the optical transitions from bulk to surface states give rise to a derivative-like structure in the ER spectra, which shifts with bias potential. Integration of this structure leads to the actual absorption band, taking a reasonable background subtraction into account. This absorption band for surface state $B$ has been shown to be surprisingly wide (about $1 \mathrm{eV}$ ) for the metal-electrolyte interface $/ 28 /$, much wider than expected to be for the metal-vacuum interface (about $0.3 \mathrm{eV} / 29 /$ ). The small value in the latter case arises from the fact that the optical transition into the surface state does not start right at the top of the bulk band, as there bulk and surface states have the same p-like symmetry /23/. With increasing photon energy deeper-lying bulk states become involved in the transition, which have admixtures of allowed character, and hence absorption is observed. The broadening of the surface state level up to $1 \mathrm{eV}$ reflects the timely fluctuations of the water dipoles in the double layer. Hence, a line shape analys is should throw some light onto the microscopic structure of the interfacial water and its potential dependence $/ 30,31 /$.

\section{4 - Contributions to the ER from the Double Layer}

The ER effect is usually dominated by contributions from the metal surface, especially in the interband transition region. The potential-induced changes in the optical properties of the Helmholtz layer proper, which constitutes a purely. dielectric film in the photon energy range under study, are expected to give rise to $\Delta R / R$ values which are orders of magnitude smaller than those of the metal surface $19 \%$. Attempts have been made in the past to unravel these two types of contributions for polycrystalline surfaces $/ 32,33 /$, but the assumptions used are likely to be wrong because of over-simplification of the metal's role in ER.

When extending the wave length range for the optical studies into the infrared region, i.e., well below the onset of interband transitions, optical effects originating from a potential modulation of the Helmholtz layer may be seen. In this region the free-electron contribution of the metal gives rise to a smooth and unstructured background only, and effects from surface states can be avoided by choosing the appropriate crystallographic surface or surface direction (e.g., $\vec{e} \| l[110]$ for $A g(110)$; see previous section). Such a situation is shown in Fig. 9b, where we find a derivative-like structure in $\Delta R / R$ right around the pzc $(-1.0 \mathrm{~V})$, which does not shift with wave length. This structure, which is observed also for the other single crystal faces of $\mathrm{Ag}$, has been investigated in more detail for the $\mathrm{Ag}$ (111) surface, because for this face the metal ER is hardly seen in the infrared. In Fig. 10, the double layer capacity of $\mathrm{Ag}(111)$ in $0.5 \mathrm{M} \mathrm{NaF}$ is shown and compared with the corresponding reflectivity change at $1000 \mathrm{~nm}$ with potential. The latter curve was obtained by integration of the ER signal $-(\Delta R / R) / \Delta U$. The close similarity between both curves led us to believe that this derivative-like structure in the ER signal is indeed originating from the Helmholtz layer. The sign of the optical response is such that the reflectivity of the interface is lowest at the pzc (more strictly speaking, at the maximum of $C_{D L}$ ) and it increases with potential on either side. This behavior is rather difficult to rationalize in a simple three-phase model with a purely dielectric film /34/. For example, at 


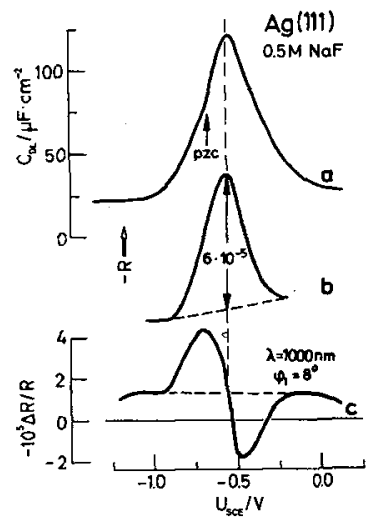

Fig. 10 - Double layer capacity (a), integrated ER signal (b, see text) and ER signal (c) for $\mathrm{Ag}$ (111) in 0.5 $\mathrm{M} \mathrm{NaF}$ as a function of potential. Normal incidence. $\lambda=1000 \mathrm{~nm}$. $\Delta U_{P P}=112 \mathrm{mV}$. pzc: $-0.67 \mathrm{~V}$.

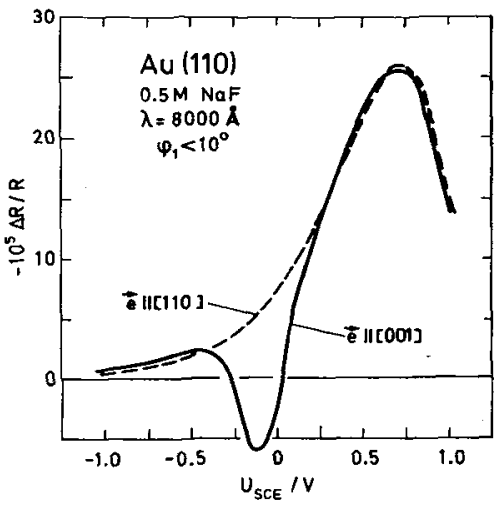

Fig. 11 - Normal-incidence ER signals for $\mathrm{Au}(110)$ in $0.5 \mathrm{M} \mathrm{NaF}$ as a function of potential. Two different directions of the electric vector. $\lambda=800 \mathrm{~nm}$. pzc: $=0.09 \mathrm{~V}$.

$1000 \mathrm{~nm}$, the effect in $\Delta R / R$ is too large to be explained solely by a change in the refractive index of the interfacial water. However, we could account for the observed effect by assuming slightly absorptive properties of the double layer water at the potential of the hump. Such a modification of the water properties, which are purely dielectric for bulk water at $1000 \mathrm{~nm}$, could arise from a specific interaction of water with the Ag surface, as invoked by Trasatti for metals with large inner-layer capacity at the pzc /35\%. An alternate explanation may be sought in the specific adsorption of fluoride which was reported by Valette /36/ to occur on Ag single crystal surfaces.

A quite different behavior was observed for gold. The potential dependence of $\Delta R / R$ for $A u(110)$ at $800 \mathrm{~nm}$ is shown in Fig. 11 for the two main crystallographic directions. There is a pronounced anisotropy around the pzc $(-0.09 \mathrm{~V})$, which is not affected in shape by a wave length change. Hence, surface states can be excluded as a cause of the anisotropy. The optical properties of the double layer seem to undergo marked changes around the pzc, which are seen only with Tight for $\overrightarrow{\mathrm{e}} \|[001]$.

\section{5 - The Role of Non-Local Optics in ER}

One of the great virtues of ER at the metal-electrolyte interface is the high sensitivity with which reflectance changes can be detected. Hence, theories on metal optics can be tested against experiment with high accuracy. It has been shown that standard optics (transverse electromagnetic waves only) should fail in describing the reflectivity of metal surfaces near the volume plasma frequency, when $\mathrm{p}$-polarized light is used $/ 37,38 /$. In this frequency region (e.g., $3.8 \mathrm{eV}$ for $\mathrm{Ag}$ ), longitudinal waves (plasma waves) are eigenmodes of the metal which are optically excited with p-polarized light because the electric field component normal to the surface induces periodic charge-density fluctuations $/ 39 /$. The longitudinal waves propagate into the metal, in addition to the transverse waves, and therefore contribute to the reflectance and transmittance of the metal surface. It has been shown for Ag that around $3.8 \mathrm{eV}$ the ER spectra for p-polarized light could not be reproduced by standard optics, unless one were willing to assume rather peculiar optical properties for the surface layer. This discrepancy was removed by using proper metal optics, i.e., by including spatial dispersion 
(non-local effects) in the calculation $/ 40,41 /$. In the following the influence of non-local effects on the ER spectra of metals is demonstrated by two examples.

When the ER spectra of Au are calculated by standard optics, using the free-electron model, a pronounced peak in $-(\Delta R / R)$ below the volume plasma frequency of gold $(2.5 \mathrm{eV})$ is predicted for p-polarization, especially for positive bias potentials (see Fig. 12b, dotted line) 142/. Such a feature has never been observed in experiment, and it is obviously an artifact arising from the use of an improper metal optics. The experimental spectra in this wave length region have about the same shape for $s$ - and p-polarized light, with a difference in magnitude of about a factor of two for $45^{\circ}$ angle of incidence $16,9 /$. When calculations with non-local optics are performed, the feature described above is no longer found in the theoretical spectrum (Fig. 12, solid line), and a spectral shape emerges which is in very good agreement with experiment $/ 43 /$. In local optics it is assumed that the surface layer has a plasma frequency of its own, which differs from the bulk plasmon frequency because of the field-induced change in the surface electron density. Charging the Au surface positively reduces the free-electron contribution to the real part of the Au dielectric constant $\varepsilon^{\prime}$ to such an extent that $\varepsilon^{\prime}$ of the surface layer becomes zero at lower photon energies /42/. The local treatment of the surface layer, for which now the plasmon resonance condition $\left(\varepsilon^{\prime}=0\right)$ is fulfilled at energies below $2.5 \mathrm{eV}$, leads to the pronounced peak in $-\Delta R / R$ around $2.3 \mathrm{eV}$ (Fig. 12b). The correct, non-local optics treats the surface charge density not as singularity but allows a spreading normal to the surface, which couples the plasmon waves of the surface layer to those of the bulk. This makes the $\Delta R / R$ values rather insensitive to the surface charge density, at least in the region of the plasma frequency, in agreement with experimental findings.

The second example refers to the ER of a thin $\mathrm{Ag}$ overlayer on $\mathrm{Au}(111) / 44 /$. In Fig. 13, $\Delta R / R$ is shown for several wave lengths around the plasma frequency of $\mathrm{Ag}$ as a function of thickness $D$ of the $A g f i l m$, which was deposited onto Au(111) under diffusion controlled conditions. We note pronounced oscillations in $\Delta R / R$ for p-polarized light, while for s-polarization a smooth curve is found (we ignore for this consideration the thickness region below $0.5 \mathrm{~nm}$ because the optical properties of the first monolayer are strongly coverage dependent and hence cause structures in $\Delta R / R$ for both polarizations). The oscillations in $\Delta R / R$ for p-polarized light are caused by the formation of standing plasma waves as the $\mathrm{Ag} f \mathrm{film}$ acquires certain multiple thickness values. These features have been reproduced by non-1ocal optics assuming bulk optical properties for the Ag-film /44/. An

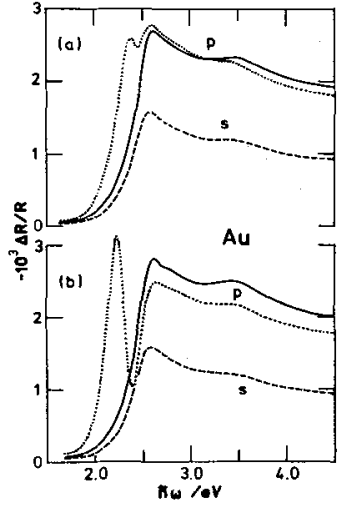

Fig. 12 - Calculated ER spectra for $\mathrm{Au}$ at $45^{\circ}$ for two different potential steps. (---) s-polarization; (-) p-polarization with plasma waves; ( $\because$.$) ) p-polarization with$ local optics. $0.4 \mathrm{~nm}$ thick surface layer; free electron density change from (a) 0.82 to 0.77 , and (b) 0.70 to 0.65 of $n_{b u l k}$ (pzc: $n=0.75$ ). After ref. $/ 43 /$.

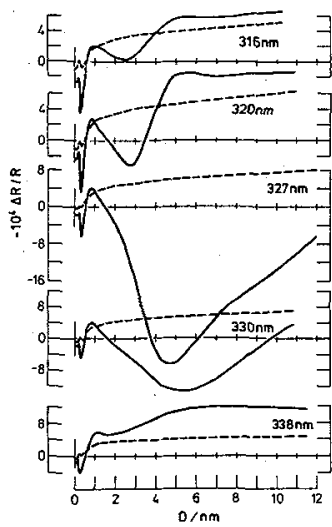

Fig. 13 - ER signals for Au(111) during deposition of an $\mathrm{Ag}$ overlayer of thickness D. $\varphi_{1}=45^{\circ}$. $(-\mathrm{p}$ - and (---) s-polarization. $\Delta U_{p P}=100 \mathrm{mV}$. After ref. $/ 44 /$. 
evaluation of the curves in Fig. 13 by classical optics clearly would be misleading as we would be forced to assume strange, oscillating optical properties of the Ag film, which is not the case.

Acknowledgement: This article was written while the author was a Visiting Scientist at the IBM Thomas J. Watson Research Center, Yorktown Heights, U.S.A.

\section{References}

11 Parsons, R., in: Comprehensive Treatise of Electrochemistry, Vol. 1, ed. by J. 0'M. Bockris, B.E. Conway and E. Yeager (Plenum Press, New York, 1980) p.1.

12/ Gerischer, H., D.M. Kolb and J.K. Sass, Adv. Phys. 27 (1978) 437.

/3/ Feinleib, J., Phys. Rev. Lett. 16 (1966) 1200.

14/ See, e.g., M. Cardona, Modulation Spectroscopy (Academic Press, New York, 1969).

15/ Lang, N.D., and W. Kohn, Phys. Rev. B7 (1973) 3541.

16/ See, e.g., J.D.E. McIntyre, Surface Sci. 37 (1973) 658.

17/ Prostak, A., and W.N. Hansen, Phys. Rev. 160 (1967) 600.

18/ Hansen, W.N., and A. Prostak, Phys. Rev. 174 (1968) 500.

19/ McIntyre, J.D.E., in: Adv. Electrochem. ETectrochem. Engin., Vol. 9, ed. by R.H. Muller (Wiley, New York, 1973) p. 61.

/10/ Furtak, T.E., and D.W. Lynch, Phys. Rev. Lett. 35 (1975) 960.

/11/ Kolb, D.M., and R. Kötz, Surface Sci. 64 (1977) 96.

/12/ Kötz, R., and D.M. Kolb, Z. Physik. chem. N.F. 112 (1978) 69.

/13/ Lynch, D.W., Surface Sci. 103 (1981) 289.

/14/ Furtak, T.E., and D.W. Lynch, Nuovo Cimento 39B (1977) 346.

/15/ Furtak, T.E., and D.W. Lynch, J. Electroanal. Chem. 79 (1977) 1.

/16/ Huong, N.V., C. Hinnen, J. LeCoeur and R. Parsons, J. Electroana 1. Chem. 92 (1978) 239; note, that the crystallographic directions for $A u(110)$ have been interchanged.

/17/ Kofman, R., R. Garrigos and P. Cheyssac, Surface Sci. 101 (1980) 231.

/18/ Tadjeddine, A., D.M. Kolb and R. Kötz, Surface Sci. 101 (1980) 277.

/19/ Kötz, R., and H.J. Lewerenz, Surface Sci. 78 (1978) [233.

/20/ Kötz, R., and D.M. Kolb, unpublished.

/21/ Smoluchowski, R., Phys. Rev. 60 (1941) 661.

/22/ Ho, K.M., B.N. Harmon and S.H. Liu, Phys. Rev. Lett. 44 (1980) 1531.

/23/ Kolb, D.M., W. Boeck, K.M. Ho and S.H. Liu, Phys. Rev. Lett. 47 (1981) 1921.

/24/ Boeck, W., and D.M. Kolb, Surface Sci. 118 (1982) 613.

/25/ Yeager, E., J. Physique 38 (1977) C5-1.

126/ Kofman, R., R. Garrigos and P. Cheyssac, Thin Solid Films 82 (1981) 73.

127/ Carnie, S.L., and D.Y.C. Chan, J. Chem. Phys. 73 (1980) $29 \overline{49}$.

128/ Ho, K.M., C.Y. Fu, S.H. Liu, D.M. Kolb and G. Piazza, J. Electroanal.

Chem., (1983) in press.

/29/ Ho, K.M., private communication.

/30/ Liu, S.H., Surface Sci. 105 (1981) 429.

/31/ MacDonald, J.R., and S.H. Liu, Surface Sci. 125 (1983) 653.

/32/ Bewick, A., and J. Robinson, J. Electroana T. Chem. 60 (1975) 163.

133/ Hinnen, C., Nguyen Van Huong, A. Rousseau and J.P. Dalbera, J. Electroanal. Chem. 95 (1979) 131.

/34/ McIntyre, J.D.E., and D.E. Aspnes, Surface Sci. 24 (1971) 417.

135/ Trasatti, S., J. Electroana1. Chem. 123 (1981) 121.

/36/ Valette, G., J. Electroana1. Chem. $\frac{138}{38}$ (1982) 37.

/37/ Forstmann, F., and R.R. Gerhardts, in: Festkörperprobleme / Adv. Solid State Phys., Vol. 22 (Vieweg, Braunschweig, 1982) p. 291.

138/ Feibelman, P.J., Progr. Surface Sci., (1983) in press.

/39/ Forstmann, F., and H. Stenschke, Phys. Rev. B17 (1978) 1489.

/40/ Kötz, R., D.M. Kolb and F. Forstmann, Surface Sci. 91 (1980) 489.

/41/ Kötz, R., and D.M. Kolb, Surface Sci. 97 (1980) 575.

/42/ McIntyre, J.D.E., and W.F. Peck, Faraday Disc. Chem. Soc. 56 (1973) 122.

/43/ Forstmann, F., K. Kempa and D.M. Kolb, J. Electroanal. Chem., (1983) in press. 144/ Piazza, G., D.M. Kolb, K. Kempa and F. Forstmann, in preparation. 